\title{
Dossiers des coroners sur la mortalité par suicide à Montréal : limites et incidences sur les stratégies de prévention du suicide
}

\author{
J. Houle, Ph. D.; C. Guillou-Ouellette, B. Sc.
}

Cet article a fait l'objet d'une évaluation par les pairs.

\section{Résumé}

Introduction : À Montréal, les caractéristiques des cas de suicide sont susceptibles de varier d'un territoire à l'autre. Les données recueillies par les coroners dans le cadre de leurs investigations sur les suicides pourraient servir à appuyer la planification en matière de prévention du suicide à l'échelle locale.

Méthodologie : Dans cette étude, nous avons analysé tous les dossiers des coroners relatifs aux décès par suicide survenus à Montréal entre 2007 et 2009 afin de déterminer l'utilité des données disponibles, d'établir un profil des cas de suicide et d'examiner les différences à l'échelle locale en comparant deux territoires, celui ayant le taux de suicide le plus élevé et celui ayant le taux le plus faible.

Résultats : Les données recueillies ont révélé qu'il n’existait pas de procédure uniforme et systématique pour la consignation des données sur les décès par suicide. Quoique les taux de données manquantes aient été variables, ils étaient très élevés en ce qui concerne les antécédents de tentative de suicide et les événements récents qui pourraient avoir provoqué le suicide. Nous avons observé des différences dans les caractéristiques des personnes qui s'enlèvent la vie en fonction du territoire de résidence.

Conclusion : L'adoption par les coroners d'une procédure uniforme de collecte de données sur les cas de suicide permettrait aux décideurs d'avoir un portrait plus juste des personnes décédées par suicide au sein de leur territoire. S’adapter à la situation locale pourrait améliorer les stratégies de prévention du suicide.

Mots-clés : suicide, coroner, prévention, surveillance

\section{Introduction}

Entre 2000 et 2009, on a observé une diminution significative du taux de suicide au Québec, qui est passé de 16,8 pour 100000 à 12,5 pour 100 000, tandis que le taux canadien demeurait relativement stable, passant de 11,4 à 10,7 pour $100000^{1,2}$. Cette baisse ne touche cependant pas tous les sous-groupes de population de la même manière ${ }^{3}$. Ainsi, la diminution du taux de suicide chez les jeunes de 15 à 19 ans a été remarquable (10\% chez les garçons et $14 \%$ chez les filles), mais chez les personnes de 50 ans et plus, le taux de suicide est demeuré relativement stable pour les deux sexes. Ce constat donne à penser qu'il convient d'améliorer les stratégies existantes de prévention du suicide chez les adultes vieillissants. Même si les stratégies universelles de prévention du suicide (p. ex. la restriction des moyens) sont efficaces ${ }^{4}$, les experts conviennent généralement qu'il faut mettre en œuvre des stratégies sélectives qui ciblent des populations à risque particulières et tiennent compte de facteurs tels que l'âge, la situation socioéconomique, les normes culturelles et le milieu social ${ }^{5-8}$. Des interventions adap- tées se sont avérées efficaces pour réduire le taux de suicide chez les adultes vieillissants $^{5,9}$, les policiers ${ }^{10}$ et les membres de la Force aérienne des États-Unis ${ }^{11,12}$.

Les taux de suicide ne sont pas les mêmes en milieu rural et en milieu urbain ${ }^{13}$. Les régions densément peuplées de Laval et de Montréal affichent les taux de suicide les plus bas de la province de Québec. En 2009, le taux de suicide dans la Région métropolitaine de Montréal (RMR) s'établissait à 10,1 pour $100000^{2,14}$. La RMR est divisée en 12 territoires gérés par un Centre de santé et de services sociaux (CSSS), chacun avec sa structure et ses services. Les CSSS sont responsables de mettre en œuvre les stratégies les plus efficaces en matière de prévention du suicide $^{15}$, en collaboration avec leurs partenaires du milieu communautaire, par exemple les organisations non gouvernementales et les médecins.

L'Agence de la santé et des services sociaux (ASSS) de Montréal fournit aux CSSS des données statistiques générales sur les taux de suicide sur leurs territoires respectifs ainsi que sur les liens entre ces taux et d'autres indicateurs ${ }^{16}$. Ces données statistiques font ressortir des différences considérables dans les taux de suicide en fonction des territoires desservis par les services de santé. Entre 2005 et 2009, le taux ajusté de mortalité par suicide était de 17,4 pour 100000 pour un CSSS du centre de Montréal, contre 5,1 pour 100000 pour un CSSS de l'ouest de la ville $^{14}$. Malgré leur utilité, ces données statistiques ne sauraient suffire pour dresser ne serait-ce qu'un profil sommaire des cas de suicide au sein de chaque territoire, parce qu'elles ne permettent pas de 
connaître les caractéristiques sociodémographiques des personnes décédées, ni les circonstances de leur décès. De plus, ce profil varie probablement d'un CSSS à l'autre, ce qui rend nécessaire l'adaptation des mesures préventives à la situation locale.

Au Québec, conformément à la Loi sur la recherche des causes et des circonstances des décès ${ }^{17}$, un coroner doit établir les causes de tous les décès survenus dans des circonstances obscures ou violentes, ce qui inclut tous les cas de suicide. Chaque suicide fait donc l'objet d'une investigation menée par l'un des 85 coroners de la province. Les coroners sont des médecins, des avocats ou des notaires; ils doivent avoir au minimum quatre années d'expérience professionnelle pour être nommés coroners à temps partiel, et huit années pour être nommés coroners permanents. Les coroners sont nommés par le gouvernement du Québec après un processus approfondi d'entrevue et sur recommandation du ministre de la Sécurité publique. Il y a 13 coroners dans la RMR, pour la plupart médecins à temps partiel (9).

Lorsqu'ils procèdent à une investigation sur un décès par suicide, les coroners sont tenus de produire un rapport d'investigation, mais ils ne reçoivent ni gabarit ni lignes directrices à cet effet. Les policiers sont souvent mis à contribution au cours de l'investigation, et les amis et les membres de la famille sont presque toujours consultés. Les coroners concluent rarement que la cause du décès est indéterminée (en 2009, seulement 2 \% des investigations ont donné lieu à une telle conclusion). On estime en outre que le taux de suicide non déclaré est si faible qu'il n'a guère d'incidence sur les conclusions tirées d'une analyse des rapports des coroners $^{18}$. Les rapports des coroners constituent donc une source d'information cruciale lorsqu'on se propose d'établir un profil des cas de suicide, même si le bureau du coroner n'a jamais fourni qu'une analyse minimale des cas dans chaque région, fondée sur le sexe, l'âge et le moyen utilisé.

Cette étude vise à passer en revue les informations relatives aux décès par suicide qui figurent dans les dossiers des coroners de la région de Montréal, afin de déterminer s'il est possible d'utiliser ces données à l'échelle locale pour surveiller les tendances en matière de suicide et appuyer l'élaboration de stratégies de prévention du suicide, d'établir un profil complet des cas de suicide entre 2007 et 2009 et d'examiner les différences à l'échelle locale dans le profil des cas de suicide en comparant deux territoires de services de santé, celui ayant le taux de suicide le plus élevé et celui ayant le taux le plus faible.

Le bureau du coroner en chef et le ministère de la Justice du Québec ont examiné et approuvé ce projet de recherche avant le début de la collecte de données.

\section{Méthodologie}

\section{Population}

Nous avons inclus tous les résidents de Montréal qui, selon les dossiers des coroners, sont décédés en 2007, en 2008 ou en 2009 et dont la cause déclarée de décès était le suicide. Montréal est le pôle économique du Québec, avec une population de près de 2 millions d'habitants aux origines ethniques et aux situations socioéconomiques variées. Le territoire de CSSS ayant le plus haut taux de suicide (territoire A) correspond au centre-ville, et c'est aussi l'un des quartiers les plus peuplés au Canada (environ 138500 résidents). Il est aussi socialement très hétérogène : il regroupe aussi bien des populations marginales, comme des itinérants, des prostitués et des toxicomanes, que des jeunes professionnels et des familles ${ }^{19}$. Le territoire de CSSS affichant le taux de suicide le plus faible (territoire B) compte environ 217000 résidents, et offre parmi les meilleures conditions de vie à Montréal (du point de vue socioéconomique). La population de ce territoire est composée en majeure partie de familles anglophones aux revenus annuels élevés comparativement aux revenus moyens à Montréal ${ }^{20}$.

\section{Sources des données}

Les données de notre étude sont tirées des dossiers complets établis pour chaque cas de suicide et conservés au bureau du coroner en chef du Québec. Une chercheure a examiné le rapport d'investigation du coroner, le rapport d'enquête officiel des policiers et, le cas échéant, la note de suicide ainsi que toute autre information pertinente. Ces dossiers, qui doivent être consultés sur place, ont fait l'objet d'une vérification ultérieure par un autre chercheur.

\section{Grille et variables pour la collecte des données}

Après avoir procédé à une première analyse des rapports d'investigation des coroners sur les décès par suicide survenus en 2009, nous avons conçu une grille de collecte de données. La grille a ensuite été révisée par une chercheure ayant de nombreuses années d'expérience en collecte d'information dans les dossiers du coroner. La grille finale de collecte de données visait à recueillir les éléments d'information suivants :

- Profil sociodémographique : sexe, âge, état matrimonial (seul ou en couple), parentalité, situation d'emploi, situation du ménage (vivant seul ou non), existence de problèmes financiers ou d'un casier judiciaire et enfin code postal du lieu de résidence (territoire de CSSS).

- Troubles mentaux : présence de psychopathologie (dépression, abus de substances, schizophrénie, trouble bipolaire, etc.).

- Événements récents : séparation conjugale ou perte d'emploi dans l'année précédant le décès.

- Manifestations suicidaires : tentatives de suicide antérieures et délai entre la tentative de suicide la plus récente et le décès par suicide; propos suicidaires ou changements de comportement indiquant une intention suicidaire avant le décès par suicide.

- Utilisation récente des services de santé : ressources d'aide professionnelle (médecin, psychologue, CSSS, etc.) consultées au cours de l'année précédant le décès.

- Circonstances entourant le décès : lieu du décès (domicile, milieu de travail, etc.), moyen utilisé, présence ou non d'une note de suicide et d'indices de planification. 


\section{Analyses statistiques}

Nous avons utilisé le logiciel statistique SPSS version $\mathrm{X}$ pour Windows (IBM, Chicago, Illinois, États-Unis). Les données ne figurant pas dans le dossier du coroner pour un cas ont été considérées comme manquantes et la fréquence des variables manquantes a été calculée pour chaque variable. Nous avons ensuite établi un profil des cas de suicide au moyen d'une analyse descriptive (fréquence, pourcentage) des données disponibles, en excluant ces données manquantes. Par exemple, nous avons calculé le pourcentage de personnes décédées par suicide qui occupaient un emploi au moment de leur décès en divisant le nombre de personnes ayant un emploi au moment de leur décès par le nombre total de personnes dont le dossier indiquait une situation d'emploi. Les différences liées au sexe ont été examinées à l'aide du test de Student pour l'âge et de tests du chi carré pour les autres variables. Enfin, nous avons eu recours à des tests du chi carré pour déterminer les différences entre les deux territoires de CSSS choisis.

\section{Résultats}

\section{Données figurant dans les dossiers des coroners}

Les données tirées des dossiers des coroners ont révélé qu'aucune procédure d'investigation uniforme n'était utilisée relativement aux décès par suicide. Le taux de données manquantes variait considérablement d'une variable à l'autre (tableau 1). Hormis les informations de base, telles que le sexe, l'âge, le lieu du suicide et le moyen utilisé - qui étaient toujours systématiquement consignées au dossier -, les autres informations pertinentes n'étaient pas systématiquement recueillies. Par exemple, les informations sur les tentatives antérieures de suicide étaient absentes dans plus de $40 \%$ des dossiers.

\section{Profil des cas de suicide à Montréal}

Au total, 566 résidents de Montréal sont décédés par suicide en 2007, 2008 ou 2009. Les résultats montrent que $74,4 \%$ des décès par suicide concernaient des

TABLEAU 1

Données relatives aux décès par suicide qui sont manquantes dans les dossiers des coroners, Montréal (Québec), 2007-2009

\begin{tabular}{|c|c|}
\hline Variable & $\begin{array}{l}\text { Données manquantes } \\
\qquad \begin{array}{c}(\mathrm{N}=566) \\
\mathbf{n}(\%)\end{array}\end{array}$ \\
\hline Sexe & $0(0,0)$ \\
\hline Âge & $0(0,0)$ \\
\hline Lieu de résidence (code postal) & $4(0,7)$ \\
\hline \multicolumn{2}{|l|}{ Profil sociodémographique } \\
\hline sans relation de couple & $16(2,8)$ \\
\hline sans emploi & $71(12,5)$ \\
\hline sans enfant & $54(9,5)$ \\
\hline vit seul(e) & $0(0,0)$ \\
\hline problèmes financiers & $250(44,2)$ \\
\hline casier judiciaire & $1(0,2)$ \\
\hline Troubles mentaux & $3(0,5)$ \\
\hline Maladies physiques & $3(0,5)$ \\
\hline \multicolumn{2}{|l|}{ Événements récents ( $\leq 1 \mathrm{an}$ ) } \\
\hline perte d'emploi & $139(24,6)$ \\
\hline séparation conjugale & $277(48,9)$ \\
\hline \multicolumn{2}{|l|}{ Manifestations suicidaires } \\
\hline tentative(s) antérieure(s) & $243(42,9)$ \\
\hline tentative(s) au cours de la dernière année & $284(50,2)$ \\
\hline propos suicidaires & $84(14,8)$ \\
\hline changements de comportement & $178(31,4)$ \\
\hline Utilisation récente (1 an ou moins) des services de santé & $0(0,0)$ \\
\hline \multicolumn{2}{|l|}{ Circonstances entourant le décès } \\
\hline note laissée & $23(4,1)$ \\
\hline indices de planification & $429(75,8)$ \\
\hline décès au domicile & $1(0,2)$ \\
\hline moyen utilisé & $0(0,0)$ \\
\hline
\end{tabular}

hommes, et que plus de la moitié $(52,3 \%)$ des personnes décédées par suicide étaient âgées de 40 à 64 ans (tableau 2). Les indices d'isolement social - le fait de ne pas vivre en couple, d'être sans emploi, de ne pas avoir d'enfant ou de vivre seul étaient fréquents. Ces indices étaient généralement cumulatifs : 55,0 \% des sujets de notre étude présentaient trois indices d'isolement social, alors que seulement $4,1 \%$ n'en présentaient aucun (données non présentées). Un grand nombre d'entre eux souffraient d'au moins un trouble mental $(63,1 \%)$, en particulier de dépression $(32,3 \%)$ et d'abus de substances (30,0 \%). La séparation conjugale était l'événement récent le plus souvent mentionné dans les dossiers des coroners (13,6 \%). Trois cas sur cinq (59,9\%) avaient consulté un professionnel pour obtenir de l'aide au cours de l'année précédant le décès, les médecins de famille $(35,7 \%)$ et les psychiatres $(27,7 \%)$ ayant été les professionnels les plus souvent consultés.

L'existence de problèmes financiers et d'un casier judiciaire était plus souvent signalée chez les hommes que chez les femmes. Les femmes étaient plus nombreuses à avoir consulté un professionnel pour obtenir de l'aide au cours de l'année précédant leur décès. Elles étaient aussi plus nombreuses à s'être enlevé la vie à leur domicile. Le moyen utilisé pour se donner la mort variait également selon le sexe : les hommes ont eu plus souvent recours à la pendaison, et les femmes à l'intoxication (tableau 2). 
TABLEAU 2

Profil des cas de suicide, Montréal (Québec), 2007-2009

\begin{tabular}{|c|c|c|c|c|}
\hline \multirow[t]{2}{*}{ Caractéristique } & Total & $\begin{array}{l}\text { Femmes } \\
(n=145)\end{array}$ & $\begin{array}{l}\text { Hommes } \\
(n=421)\end{array}$ & Valeur $p$ \\
\hline & n (\%) & n (\%) & n (\%) & \\
\hline \multicolumn{5}{|l|}{ Âge (ans) } \\
\hline 15 à 19 & $18(3,2)$ & $5(3,4)$ & $13(3,1)$ & 0,831 \\
\hline 20 à 29 & $79(13,9)$ & $19(13,1)$ & $60(14,3)$ & 0,731 \\
\hline 30 à 39 & $95(16,8)$ & $22(15,3)$ & $73(17,3)$ & 0,547 \\
\hline 40 à 49 & $142(25,1)$ & $35(24,1)$ & $107(25,4)$ & 0,760 \\
\hline 50 à 64 & $154(27,2)$ & $42(28,9)$ & $112(26,6)$ & 0,581 \\
\hline 65 et plus & $78(13,8)$ & $22(15,2)$ & $56(13,3)$ & 0,573 \\
\hline \multicolumn{5}{|l|}{ Profil sociodémographique } \\
\hline sans relation de couple & $398(70,3)$ & $102(70,3)$ & $296(70,3)$ & 0,994 \\
\hline sans emploi & $334(59,0)$ & $91(62,8)$ & $243(57,7)$ & 0,900 \\
\hline sans enfant & $296(52,3)$ & $63(43,4)$ & $233(55,3)$ & 0,001 \\
\hline vit seul(e) & $279(49,3)$ & $71(48,9)$ & $208(49,4)$ & 0,927 \\
\hline problèmes financiers & $202(35,7)$ & $37(25,5)$ & $165(39,2)$ & 0,001 \\
\hline casier judiciaire & $112(19,8)$ & $14(9,7)$ & $98(23,3)$ & 0,000 \\
\hline \multicolumn{5}{|l|}{ Troubles mentaux } \\
\hline au moins un trouble mental & $357(63,1)$ & $101(69,7)$ & $256(60,1)$ & 0,038 \\
\hline dépression & $183(32,3)$ & $56(38,6)$ & $127(30,2)$ & 0,058 \\
\hline abus de substances & $170(30,0)$ & $40(27,6)$ & $130(30,9)$ & 0,464 \\
\hline trouble bipolaire & $51(9,0)$ & $22(15,2)$ & $29(6,9)$ & 0,003 \\
\hline schizophrénie & $48(8,5)$ & $13(9,0)$ & $35(8,3)$ & 0,803 \\
\hline \multicolumn{5}{|l|}{ Événements récents ( $\leq 1 \mathrm{an}$ ) } \\
\hline séparation conjugale & $77(13,6)$ & $16(11,0)$ & $61(14,5)$ & 0,147 \\
\hline perte d'emploi & $51(9,0)$ & $8(5,5)$ & $43(10,2)$ & 0,082 \\
\hline \multicolumn{5}{|l|}{ Manifestations suicidaires } \\
\hline tentative antérieure & $208(36,7)$ & $72(49,7)$ & $136(32,3)$ & 0,081 \\
\hline tentative au cours de la dernière année & $91(16,1)$ & $31(21,4)$ & $60(14,3)$ & 0,583 \\
\hline propos suicidaires & $304(53,7)$ & $85(58,6)$ & $219(52,0)$ & 0,185 \\
\hline changements de comportement & $271(47,8)$ & $67(46,2)$ & $204(48,5)$ & 0,081 \\
\hline \multicolumn{5}{|c|}{ Utilisation récente ( $\leq 1$ an) des services de santé } \\
\hline au moins 1 service & $339(59,9)$ & $105(72,4)$ & $234(55,6)$ & 0,000 \\
\hline médecin de famille & $202(35,7)$ & $63(43,4)$ & $139(33,0)$ & 0,024 \\
\hline psychiatre & $157(27,7)$ & $59(40,7)$ & $98(23,3)$ & 0,000 \\
\hline psychologue & $23(4,1)$ & $10(6,9)$ & $13(3,1)$ & 0,045 \\
\hline \multicolumn{5}{|l|}{ Circonstances entourant le décès } \\
\hline note laissée & $246(43,5)$ & $72(49,7)$ & $174(41,3)$ & 0,110 \\
\hline indices de planification & $117(20,7)$ & $39(26,9)$ & $78(18,5)$ & 0,461 \\
\hline décès au domicile & $381(67,3)$ & $110(75,9)$ & $271(64,4)$ & 0,012 \\
\hline \multicolumn{5}{|l|}{ Moyen utilisé } \\
\hline strangulation & $259(45,7)$ & $41(28,3)$ & $218(51,8)$ & 0,000 \\
\hline intoxication & $130(22,9)$ & $61(42,1)$ & $69(16,4)$ & 0,000 \\
\hline chute & $44(7,8)$ & $13(9,0)$ & $31(7,4)$ & 0,534 \\
\hline arme à feu & $26(4,6)$ & $6(4,1)$ & $20(4,8)$ & 0,761 \\
\hline noyade & $20(3,6)$ & $6(4,1)$ & $14(3,3)$ & 0,648 \\
\hline métro & $19(3,4)$ & $4(2,8)$ & $15(3,6)$ & 0,643 \\
\hline autre & $68(12,0)$ & $14(9,6)$ & $54(12,7)$ & 0,311 \\
\hline
\end{tabular}

Comparaison entre le territoire de CSSS ayant le taux de suicide le plus faible et celui ayant le taux le plus élevé

Par rapport aux personnes décédées par suicide du territoire $\mathrm{A}$, celles du territoire B souffraient moins d'isolement social : elles étaient moins nombreuses à vivre seules et plus nombreuses à être mariées ou à vivre en couple et à avoir des enfants (tableau 3). Il était en outre plus rare que ces personnes aient un casier judiciaire ou des problèmes financiers récents ou aient déjà tenté de se suicider par le passé. Le moyen utilisé pour mettre fin à ses jours différait également : les personnes décédées par suicide du territoire A avaient plus souvent eu recours à l'intoxication, celles du territoire B à la strangulation.

\section{Analyse}

Dans cette étude, nous avons analysé tous les dossiers des coroners relatifs aux décès par suicide à Montréal de 2007 à 2009 inclusivement. À l'aide d'une grille d'extraction de données, nous avons analysé 566 dossiers à la recherche de données sur le suicide. En plus de permettre d'établir un profil de toutes les personnes décédées par suicide, ce processus a révélé l'absence de procédure uniforme et systématique susceptible d'être utilisée par les coroners pour recueillir l'information sur les décès par suicide. Notre étude visait à déterminer la possibilité que les dossiers des coroners soient une source d'information valide et utile à des fins de surveillance des suicides et de planification des mesures de prévention du suicide à l'échelle locale. En raison du taux élevé de données manquantes et du manque d'uniformité dans les pratiques des coroners, nous ne pouvons recommander l'utilisation de ces dossiers à cette fin.

De nombreuses autres études - au Québec $^{21}$, au Canada ${ }^{22}$ et aux ÉtatsUnis $^{23}$ - ont souligné le manque d'exhaustivité des informations recueillies par les coroners. Bon nombre de facteurs pourraient expliquer l'ampleur des données manquantes. Tout d'abord, il n'existe pas de méthode uniforme pour la rédaction des rapports et la collecte des données à 
TABLEAU 3

Profil des cas de suicide au sein des territoires de CSSS A et B, Montréal, Québec, 2007-2009

\begin{tabular}{|c|c|c|c|}
\hline Caractéristique & $\begin{array}{c}\text { Territoire A } \\
\begin{array}{c}(\mathbf{n}=\mathbf{8 5}) \\
\mathbf{n}(\%)\end{array}\end{array}$ & $\begin{array}{c}\text { Territoire B } \\
\begin{array}{c}(\mathbf{n}=32) \\
\mathbf{n}(\%)\end{array}\end{array}$ & Valeur $p$ \\
\hline Sexe (masculin) & $64(75)$ & $23(72)$ & 0,706 \\
\hline \multicolumn{4}{|l|}{ Âge (ans) } \\
\hline 15 à 64 & $74(87)$ & $24(75)$ & 0,115 \\
\hline 65 et plus & $11(13)$ & $8(25)$ & 0,113 \\
\hline \multicolumn{4}{|l|}{ Profil sociodémographique } \\
\hline sans relation de couple & $70(82)$ & $15(47)$ & 0,000 \\
\hline sans emploi & $48(57)$ & $18(56)$ & 0,956 \\
\hline sans enfant & $61(72)$ & $13(41)$ & 0,001 \\
\hline vit seul(e) & $53(62)$ & $9(28)$ & 0,001 \\
\hline problèmes financiers & $35(41)$ & $4(13)$ & 0,005 \\
\hline casier judiciaire & $20(24)$ & $2(6)$ & 0,033 \\
\hline \multicolumn{4}{|l|}{ Troubles mentaux } \\
\hline au moins 1 trouble mental & $55(65)$ & $16(50)$ & 0,145 \\
\hline dépression & $27(32)$ & $12(38)$ & 0,557 \\
\hline abus de substances & $31(37)$ & $2(6)$ & 0,001 \\
\hline trouble bipolaire & $5(6)$ & $1(3)$ & 0,547 \\
\hline schizophrénie & $12(14)$ & $1(3)$ & 0,092 \\
\hline \multicolumn{4}{|l|}{ Manifestations suicidaires } \\
\hline tentative antérieure & $44(52)$ & $8(25)$ & 0,001 \\
\hline tentative au cours de la dernière année & $20(24)$ & $2(6)$ & 0,085 \\
\hline propos suicidaires & $49(58)$ & $21(66)$ & 0,667 \\
\hline changements de comportement & $35(41)$ & $16(19)$ & 0,874 \\
\hline \multicolumn{4}{|c|}{ Utilisation récente (au cours de la dernière année) des services de santé } \\
\hline au moins 1 service & $53(62)$ & $17(53)$ & 0,364 \\
\hline médecin de famille & $30(35)$ & $11(34)$ & 0,926 \\
\hline psychiatre & $22(26)$ & $8(25)$ & 0,922 \\
\hline psychologue & $6(7)$ & $3(9)$ & 0,675 \\
\hline \multicolumn{4}{|l|}{ Circonstances entourant le décès } \\
\hline note laissée & $40(47)$ & $11(34)$ & 0,181 \\
\hline indices de planification & $12(14)$ & $9(28)$ & 0,754 \\
\hline décès au domicile & $63(74)$ & $21(66)$ & 0,363 \\
\hline \multicolumn{4}{|l|}{ moyen utilisé } \\
\hline strangulation & 32 (38) & $15(47)$ & 0,364 \\
\hline intoxication & $32(38)$ & $4(13)$ & 0,009 \\
\hline chute & $12(14)$ & $0(0)$ & 0,025 \\
\hline arme à feu & $2(2)$ & $3(9)$ & 0,094 \\
\hline noyade & $0(0)$ & $2(6)$ & 0,020 \\
\hline
\end{tabular}

Abréviation : CSSS, Centre de santé et de services sociaux.

verser au dossier officiel. Par conséquent, certains coroners s'intéressent avant tout aux causes de suicide, tandis que d'autres mettent fin à l'investigation dès qu'ils ont déterminé si la cause du décès était intentionnelle, accidentelle ou liée à un homicide. Enfin, au Québec, en raison de

ques, il est difficile pour les coroners d’obtenir des données médicales importantes, notamment celles ayant trait à un diagnostic de troubles mentaux ou à une hospitalisation à la suite d'une tentative de suicide.
Les données recueillies par les coroners dans le cadre de leurs investigations pourraient pourtant se révéler extrêmement utiles dans la prévention du suicide. Les coroners ont en effet un accès direct et privilégié à la famille du défunt et à d'autres sources d'information, comme le rapport de police, le rapport des analyses toxicologiques et le dossier médical. Ces sources d'information pourraient nous aider à mieux comprendre les circonstances entourant les décès par suicide et à établir un profil des cas de suicide susceptible de guider la prise de décision en matière de prévention du suicide. Malheureusement, les dossiers des coroners sont souvent des sources incomplètes d'information. Dans près de la moitié des dossiers, il n'existe aucune information sur les tentatives antérieures de suicide ou sur les événements récents qui pourraient avoir provoqué le suicide, tels qu'une séparation conjugale ou la perte d'un emploi. En revanche, les diagnostics de troubles mentaux et les antécédents d'utilisation des services de santé sont toujours consignés dans les dossiers des coroners. Cette situation pourrait s'expliquer par le fait que les coroners adoptent un modèle biomédical, selon lequel le suicide est considéré comme une complication d'une maladie mentale $^{24}$. Comme nous l'avons précisé plus haut, la plupart des coroners de Montréal sont médecins. Toutefois, même si les coroners s'intéressent toujours aux troubles mentaux, ils semblent sous-estimer la prévalence de ces derniers. En effet, moins des deux tiers $(63,1 \%)$ des dossiers mentionnaient au moins un trouble mental, alors que, dans les autopsies psychologiques, ce pourcentage se situe entre $80 \%$ et $96 \%{ }^{25,26}$. Le même constat s'applique à l'utilisation des services de santé dans l'année précédant le suicide : selon les dossiers des coroners, $36 \%$ des personnes décédées par suicide avaient consulté un omnipraticien au cours de l'année précédant leur décès, alors que ce pourcentage se situe entre $76 \%$ et $86 \%$ dans des études rigoureuses sur la question $^{27,28}$.
Une grille uniforme de collecte de données, qui aborderait tous les paramètres à prendre en compte dans la prévention du suicide, aiderait à réduire l'ampleur des 
données manquantes dans les dossiers des coroners. Aux États-Unis, les Centers for Disease Control and Prevention (CDC) ont parrainé la création du National Violent Death Reporting System (NVDRS) ${ }^{29,30}$. Ce système de surveillance active à l'échelle de chaque État recueille des données sur les facteurs de risque relativement à tous les décès dans des circonstances violentes, ce qui inclut les homicides, les suicides et les décès accidentels par arme à feu. On utilise l'information détaillée enregistrée dans le système pour concevoir, mettre en œuvre et évaluer des stratégies visant à réduire et à prévenir les décès dans des circonstances violentes. Les circonstances à l'origine du suicide font l'objet d'un examen particulièrement attentif (par exemple, les diagnostics et traitements de troubles mentaux, les problèmes d'abus de substances, les problèmes interpersonnels avec son partenaire, le décès récent d'un membre de la famille ou d'un ami, les problèmes financiers, la violence interpersonnelle, etc. $)^{31}$. Cet instrument pourrait s'avérer extrêmement précieux dans l'élaboration de grilles d'investigation plus complètes et mieux structurées à l'intention des coroners au Québec.

L'amélioration de la qualité et de l'exhaustivité des données recueillies par les coroners ne garantira pas pour autant leur utilisation par les décideurs locaux, qui n’ont pas actuellement accès à cette information. Afin de surmonter ce problème, on a entrepris de mettre sur pied un observatoire régional des tentatives de suicide et des suicides à Montréal. Suicide Action Montréal et le Centre de recherche et d'intervention sur le suicide et l'euthanasie (CRISE) de l'Université du Québec à Montréal seront conjointement responsables de cet observatoire. Ce dernier aura accès à toutes les données disponibles sur les personnes décédées par suicide (dont les données du bureau du coroner et les données administratives sur la facturation des médecins et sur l'hospitalisation). Ces données seront anonymisées et stockées de manière sécuritaire afin de protéger les renseignements personnels. Une équipe de chercheurs sera chargée de produire régulièrement des profils locaux pertinents à l'intention des décideurs et des responsables de la planification du programme. Il y a tout lieu de croire que ces renseigne- ments, conjugués à l'infrastructure en place axée sur l'utilisation des données recueillies par les coroners, permettront d'améliorer les stratégies ciblées de prévention du suicide.

La variance importante observée dans les taux de suicide au sein des 12 territoires de CSSS à Montréal est sans aucun doute en partie attribuable à la grande hétérogénéité sociale de cette ville. Notre étude se distingue par le fait que nous avons réussi à établir des profils différents des cas de suicide dans deux territoires de CSSS : dans le territoire A, qui présente le plus haut taux de suicide, les personnes qui s'enlèvent la vie sont souvent isolées socialement et ont un problème d'abus de substances, tandis que dans le territoire B, qui affiche le taux de suicide le plus faible, un plus grand nombre de ces personnes semblent bien intégrées socialement, et le taux d'abus de substances est faible. Cela étant dit, ces données doivent être interprétées avec prudence en raison du petit nombre de cas $(n=117)$, en particulier au sein du territoire ayant le taux de suicide le plus faible $(\mathrm{n}=32$ ). Ces constats n'en indiquent pas moins que l'on ne pourra améliorer l'efficacité des stratégies de prévention du suicide que si des mesures préventives distinctes sont prises pour ces deux sous-populations. Si les équipes de santé mentale des CSSS connaissaient mieux les caractéristiques des cas de suicide au sein de leur territoire, elles pourraient adapter leurs interventions en conséquence, par exemple en exerçant une surveillance plus étroite des sujets qui présentent un profil de risque particulier.

\section{Points forts et limites}

Notre analyse des dossiers des coroners a enrichi notre compréhension des cas de suicide à Montréal entre 2007 et 2009. L'originalité de notre étude tient au fait que nous avons non seulement mis en évidence les lacunes du processus d'investigation des décès par suicide, mais également établi les retombées positives potentielles associées à l'accès à des données locales détaillées et valides.

Plusieurs éléments restreignent toutefois les conclusions que nous pouvons tirer de cette étude. Premièrement, en raison du fait que nous avons utilisé les dossiers des coroners comme unique source de données, les résultats sont limités par la qualité inégale des informations compilées et par l'absence de certaines informations importantes. Pour être en mesure d'établir un profil plus complet et plus précis, il aurait fallu effectuer des autopsies psychologiques. Cette méthode de recherche consiste à mener des entrevues structurées auprès des membres de la famille et des amis des personnes qui se sont donné la mort, afin de déterminer avec précision l'état physique et mental de la personne au moment du décès et d'examiner les circonstances qui ont conduit au décès. Si nous avions utilisé les bases de données administratives de la Régie de l'assurance maladie du Québec, nous aurions aussi pu décrire avec certitude l'utilisation que la personne avait faite des ressources médicales au cours de l'année précédant son décès. Nous aurions également pu confirmer ou ajouter certains diagnostics de problèmes de santé mentale. Pour des raisons de faisabilité et en raison du caractère exploratoire de cette étude, nous avons restreint l'analyse à une période de trois ans. Enfin, l'interprétation des résultats au sein des territoires qui ont connu peu de suicides au cours de cette période doit être effectuée avec une grande prudence.

\section{Conclusion}

Les décisions relatives à la mise en œuvre des mesures de prévention du suicide sont presque toutes prises à l'échelle locale. Les CSSS jouent un rôle clé à cet égard, en mettant en place des services à l'intention de leur clientèle et en veillant à coordonner leurs interventions avec celles de tous leurs partenaires dans la collectivité. Afin de jouer efficacement ce rôle, les CSSS ont besoin de données détaillées sur les cas de suicide au sein de leur territoire. Avec ses 12 CSSS, Montréal a une population très hétérogène. Notre étude a montré que cette diversité se traduit également par une variabilité géographique du profil des cas de suicide, ce qui fait que les profils généraux relatifs à l'ensemble de la population montréalaise ne sont guère utiles pour les décideurs : nous ne devons pas nous en tenir à ces constatations 
générales, mais bien plutôt leur fournir de l'information plus précise.

En raison de l'ampleur du problème du suicide et de ses conséquences tragiques pour des milliers de Canadiens chaque année, il importe de prendre les mesures les plus énergiques possible. Les coroners ont un rôle important à jouer dans la réduction des taux de suicide, car ils peuvent nous aider à mieux comprendre les causes de ce phénomène. Ils pourraient grandement contribuer à l'avancement des connaissances dans ce domaine en se dotant d'une procédure uniforme et systématique de collecte d'informations sur les décès par suicide. Ces connaissances pourraient conduire à offrir des interventions mieux ciblées et plus efficaces auprès des personnes à risque.

\section{Remerciements}

La réalisation de cette étude a été rendue possible grâce au soutien financier de Suicide Action Montréal et du Centre de recherche et d'intervention sur le suicide et l'euthanasie (CRISE). Les auteures tiennent à remercier Carole Renaud, qui a effectué la collecte de données au bureau du coroner à Québec, Francis Allard qui a procédé à la vérification de l'ensemble des résultats, Paul-André Perron du bureau du coroner en chef ainsi que Philippe Angers et Véronique Landry de Suicide Action Montréal qui ont lu le rapport et fait part de leurs commentaires. Nos remerciements vont également à Charles Cardinal, bibliothécaire au Centre de recherche et d'intervention sur le suicide et l'euthanasie, pour son aide précieuse dans le cadre de la recension des écrits.

\section{Références}

1. Statistique Canada. Mortalité : liste sommaire des causes, 2000. Statistique Canada, Division de la statistique sur la santé. Ottawa (Ont.) : Statistique Canada. 2006. [n ${ }^{\circ}$ 84F0209X au catalogue]

2. Statistique Canada. Mortalité : liste sommaire des causes, 2009. Statistique Canada, Division de la statistique sur la santé. Ottawa (Ont) : Statistique Canada; 2012. [no 84F0209X au catalogue]
3. Gagné $M$, Saint-Laurent D. La mortalité par suicide au Québec : tendances et données récentes, 1981 à 2008. Direction recherche, formation et développement. Québec (Qc) : Institut national de santé publique; 2012. 19 p. [INSPQ, $\mathrm{n}^{\mathrm{o}}$ de publication : 890]

4. Mann JJ, Apter A, Bertolote J et collab. Suicide prevention strategies: a systematic review. JAMA. 2005 Oct;294(16):2064-74.

5. Erlangsen A, Nordentoft $\mathrm{M}$, Conwell $\mathrm{Y}$ et collab. Key considerations for preventing suicide in older adults. Crisis. 2011;32(2): 106-9.

6. Davis SP, Arnette NC, Bethea KS et collab. The Grady Nia Project: A culturally competent intervention for low-income, abused, and suicidal African American women. Prof Psychol Res Pr. 2009:40(2):141-7.

7. Nordentoft M. Crucial elements in suicide prevention strategies. Prog Neuropsychopharmacol Biol Psychiatry. 2011;35(4): 848-53.

8. Schwartz-Lifshitz M, Zalsman G and Giner L. Can we really prevent suicide? Curr Psychiatry Rep. 2012;14(6):624-33.

9. De Leo D, Dello Buono MD, Dwyer J. Suicide among the elderly: the long-term impact of a telephone support and assessment intervention in northern Italy. Br J Psychiatry. 2002;181:226-9.

10. Mishara BL, Martin N. Effects of a comprehensive police suicide prevention program. Crisis. 2012;33(3):162-8.

11. Knox KL, Litts DA, Talcott GW, Feig JC, Caine ED. Risk of suicide and related adverse outcomes after exposure to a suicide prevention programme in the US Air Force: cohort study. BMJ. 2003; 327:1376.

12. Knox KL, Pflanz S, Talcott GW et collab. The Air Force suicide prevention program: implications for public health policy. Am J Public Health. 2010;100(12):2457-63.

13. Ostry AS. The mortality gap between urban and rural Canadians: a gendered analysis. Rural Remote Health. 2009;9(4):1286.
14. Direction de la santé publique de Montréal. Taux de mortalité par suicide, Montréal, CSSS et CLSC, 2005-2009. Montréal (Qc) : Direction de la santé publique de Montréal; 2009.

15. Lane J, Archambault J, Collins-Poulette M, Camirand R. Prévention du suicide : guide des bonnes pratiques à l'intention des intervenants des centres de santé et de services sociaux. Québec (QC) : Direction des communications, ministère de la Santé et des Services sociaux; 2010.

16. Agence de la santé et des services sociaux de Montréal. Regard local sur la défavorisation et le suicide. Présentation dans le cadre des ateliers de gestionnaires sur l'implantation du Guide de bonnes pratiques en prévention du suicide à l'intention des gestionnaires des Centres de santé et de services sociaux et des réseaux locaux de services. Montréal (Qc) : Agence de la santé et des services sociaux de Montréal; 2012.

17. Loi sur la recherche des causes et des circonstances des décès. LRQ, 1983. c. 4, a. 2.

18. St-Laurent D, Bouchard C. L'épidémiologie du suicide au Québec : que savons-nous de la situation récente? [Internet]. Québec (QC) : Institut national de santé publique du Québec; 2004. PDF (601,42 Ko) téléchargeable à partir du lien : http://www. inspq.qc.ca/pdf/publications/283-Feuillet EpidemioSuicide.pdf

19. Guindon M. Quartiers à la loupe : un portrait pour l'action. Portrait de la population du territoire du CSSS Jeanne-Mance. Montréal (Qc) : Centre de santé et de services sociaux; 2009, 24 p.

20. Centre de santé et de services sociaux de l'Ouest-de-l'île. Portrait du CSSS de l'Ouestde-l'Île. Pointe-Claire (Qc) : Centre de santé et de services sociaux de l'Ouest-de-l'île; 2010. PDF (671 Ko) téléchargeable à partir du lien : http://www.csssouestdelile.qc.ca /fileadmin/csss_odi/publications/Portrait_ du_CSSS/brochureMars2010_portrait_CSSS. pdf

21. Boileau JC, Corriveau-Durand S, Grondines L, Lamoureux-Auclair A, Morin-Ben Abdallah S. Analyse des rapports des coroners des décès par suicide en Estrie : profils et recommandations. Centre de santé et de services sociaux, Institut universitaire de gériatrie de Sherbrooke. Sherbrooke (Qc); 2011. 
22. Campbell LA, Jackson L, Bassett $\mathrm{R}$ et collab. Recherche sur la surveillance et la prévention du suicide en Nouvelle-Écosse : pertinence de l'utilisation des dossiers des médecins légistes? Maladies chroniques et blessures au Canada. 2011;31(4):165-176.

23. Powell V, Barber CW, Hedegaard $\mathrm{H}$ et collab. Using NVDRS data for suicide prevention: promising practices in seven states. Inj Prev. 2006;12(Suppl II): ii28-32.

24. Mishara BL, Chagnon F. Understanding the relationship between mental illness and suicide and the implications for suicide prevention. Dans : O'Connor RC, Platt S, Gordon J (dir.). International handbook of suicide prevention: research, policy and practice. Chichester (UK) : John Wiley \& Sons Ltd; 2011. p. 609-623.

25. Canavagh JT, Carson AJ, Sharpe M, Lawrie SM. Psychological autopsy studies of suicide: a systematic review. Psychol Med. 2003;33(3):395-405.

26. Arsenault-Lapierre G, Kim C, Turecki G. Psychiatric diagnoses in 3275 suicides: a meta-analysis. BMC Psychiatry. 2004;4:37.

27. Luoma JB, Martin CE, Pearson JL. Contact with mental health and primary care providers before suicide: a review of the evidence. Am J Psychiatry. 2002;159(6): 909-16.

28. Morrison KB, Laing L. Utilisation des services de santé l'année avant le suicide chez les adultes en Alberta. Rapport sur la santé. 2011;22(3):17-25. [Statistique Canada, $n^{\circ}$ 82-003-XPF au catalogue]

29. Paulozzi LJ, Mercy J, Frazier L Jr, Annest JL. CDC's National Violent Death Reporting System: background and methodology. Inj Prev. 2004;10(1):47-52.

30. Steencamp M, Frazier L, Lipskiy $\mathrm{N}$ et collab. The National Violent Death Reporting System: an exciting new tool for public health surveillance. Inj Prev. 2006; 12(Suppl 2):ii3-5.
31. CDC. National Violent Death Reporting System (NVDRS) Coding Manual Version 3 [Internet]. National Center for Injury Prevention and Control, Centers for Disease Control and Prevention; 2008. PDF $(896,14$ Ko) téléchargeable à partir du lien : http://www.cdc.gov/ncipc/pub-res /nvdrs-coding/vs3/nvdrs_coding_manual_ version_3-a.pdf 